Onkologe $2021 \cdot 27: 1004-1010$

https://doi.org/10.1007/s00761-021-01006-6

Angenommen: 12. Juli 2021

Online publiziert: 3 . August 2021

๑) Springer Medizin Verlag GmbH, ein Teil von

Springer Nature 2021

\section{Qualitätssicherung bei hämatoonkologischen Schwerpunktpraxen}

\section{Ergebnisse der Strukturdatenerhebung 2019}

Kerstin Hermes-Moll · Thomas Walawgo · Sandra Osburg · Vitali Heidt

Wissenschaftliches Institut der Niedergelassenen Hämatologen und Onkologen (WINHO), Köln, Deutschland

\section{In diesem Beitrag}

- Qualitätsberichterstattung

- Strukturmerkmale hämatoonkologischer Praxen

- Strukturdatenerhebung 2019

- Verwendete Qualitätsmanagementsysteme und Zertifizierungen

- Beteiligungen an Tumorkonferenzen und klinischen Studien

- Diskussion

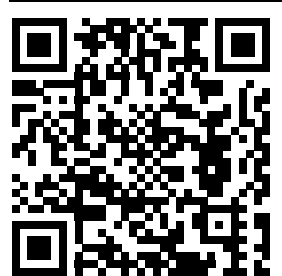

QR-Code scannen \& Beitrag online lesen

Zusammenfassung

Hintergrund: Die regelmäßig erscheinenden Qualitätsberichte der hämatoonkologischen Schwerpunktpraxen umfassen primär Abrechnungsdaten und Ergebnisse von Patienten- und Mitarbeiterbefragungen sowie aktuelle projektbezogene Ergebnisse. Um darüberhinausgehende Informationen zur Strukturqualität zu erlangen, wurde eine zusätzliche Erhebung bei den hämatoonkologischen Praxen durchgeführt. Ziel der Arbeit: Ziel waren Analyse und Darstellung struktureller Qualitätsmerkmale der hämatoonkologischen Schwerpunktpraxen.

Material und Methode: Es erfolgte eine Online-Befragung der Praxen mit mindestens einem Mitglied im Berufsverband der Niedergelassenen Hämatologen und Onkologen in Deutschland im Jahr 2019 zu Praxismerkmalen, berufspolitischen Themen sowie Qualitätsmanagement und Vernetzung.

Ergebnisse: Von 372 angeschriebenen Praxen haben sich 130 an der Strukturdatenerhebung beteiligt (35\%). Hinsichtlich der Themen Qualitätsmanagement und Vernetzung zeigen die Daten, dass über $60 \%$ der Praxen ein zertifiziertes Qualitätsmanagementsystem haben. Fast alle Praxen (94\%) sind mit mindestens einem zertifizierten Zentrum vertraglich verbunden und nehmen regelmäßig an Tumorkonferenzen teil (98\%). An klinischen Studien nehmen $77 \%$ der Praxen teil. Schlussfolgerungen: Die Ergebnisse der Strukturdatenerhebung 2019 liefern Einblicke in Qualitätsmerkmale der hämatoonkologischen Schwerpunktpraxen und belegen eine starke sektorenübergreifende Vernetzung dieser Praxen in Deutschland.

\title{
Schlüsselwörter
}

Qualitätsmanagement · Vernetzung · Zertifizierung · Tumoren · Ambulante Patienten

Für eine bundesweit flächendeckende und qualitätsgesicherte Versorgung aller an Krebs erkrankten Personen ist ein multidisziplinäres und sektorenübergreifendes Netzwerk erforderlich. Durch die Zunahme der ambulanten Versorgung rücken Themen der Qualitätssicherung und -berichterstattung bei niedergelassenen Hämatoonkologinnen und Hämatoonkologen wieder stärker in den Fokus. Die Auswertung einer Befragung zu strukturellen Qualitätsmerkmalen der hämatoonkologischen Schwerpunktpraxen spiegelt deutlich die starke Vernetzung der Praxen und die sektoren- übergreifenden Versorgungsstrukturen wider.

\section{Qualitätsberichterstattung}

In den vergangenen 3 Jahrzehnten hat sich der Blick auf die Qualitätssicherung in der ambulanten hämatoonkologischen Versorgung stark verändert [1, 2, 4]. Angesichts der Zunahme der ambulanten Versorgung in der Krebstherapie, insbesondere bei der medikamentösen Behandlung, bleiben Themen der Qualitätssicherung und des Qualitätsmanagements (QM) im ambulanten Sektor zentrale 


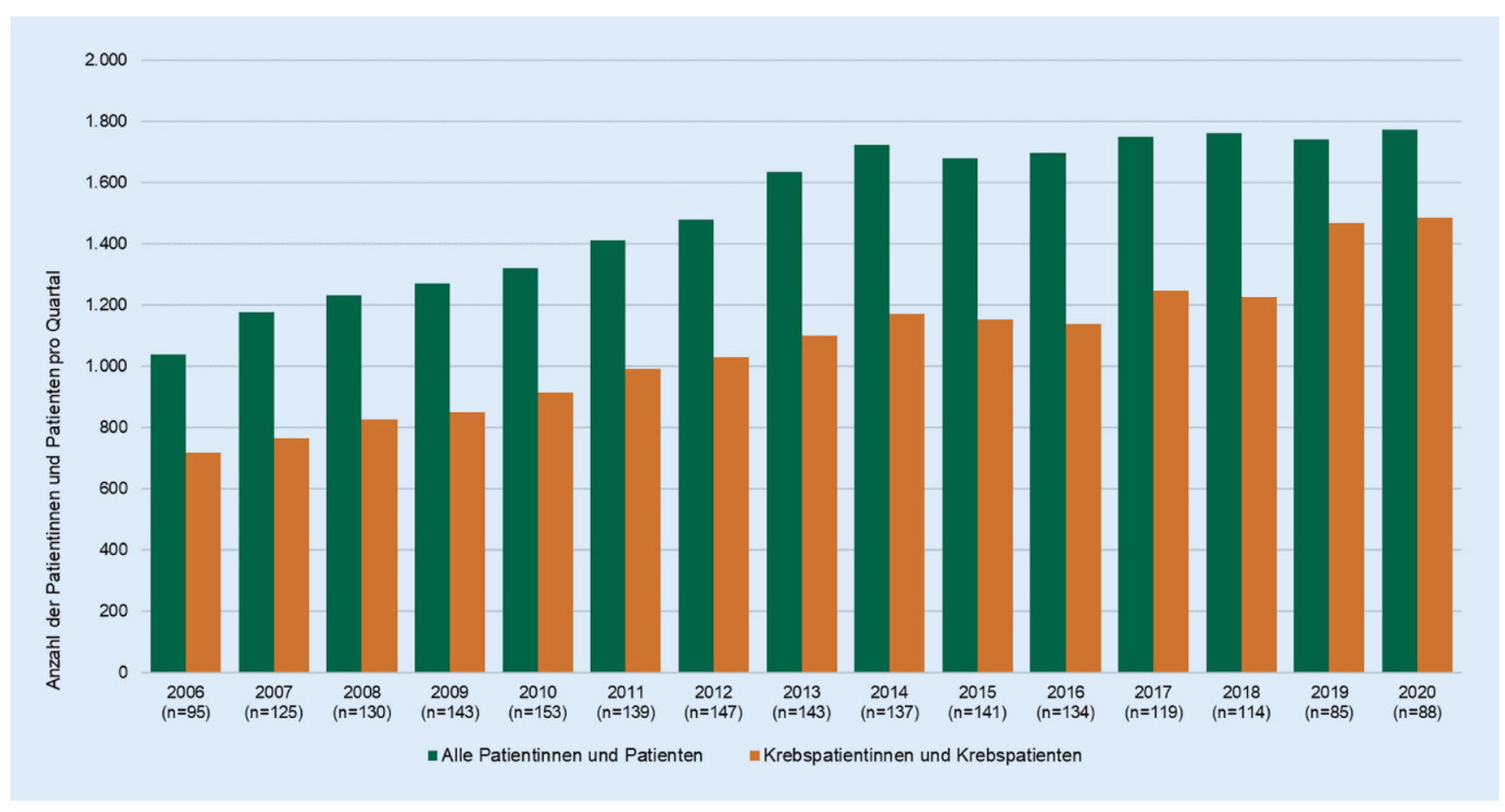

Abb. 1 A Entwicklung der durchschnittlichen Patientenzahl in hämatoonkologischen Schwerpunktpraxen in einem Quartal. Die Angaben beziehen sich jeweils auf das 3. Quartal eines Jahres. KVDT Kassenärztliche-Vereinigung-Datentransfer. (Quelle: KVDT-Daten der hämatoonkologischen Schwerpunktpraxen)

Bestandteile gesundheitspolitischer und wissenschaftlicher Betrachtungen [1, 3, 10]. Den hämatoonkologischen Schwerpunktpraxen steht ein breites Spektrum an externen und internen qualitätssichernden Maßnahmen zur Verfügung $[4,7,18]$. Die Einführung und Weiterentwicklung eines einrichtungsinternen QM ist bereits seit 2004 für alle an der vertragsärztlichen Versorgung teilnehmenden Leistungserbringer nach $\S 135$ a des Fünften Sozialgesetzbuchs (SGB V) verpflichtend. Der vom Gesetzgeber beauftragte Gemeinsame Bundesausschuss (G-BA) hat dazu die grundsätzlichen Anforderungen in der QM-Richtlinie für die vertragsärztliche Versorgung bestimmt [9]. Doch anders als in der stationären Versorgung mit den strukturierten Qualitätsberichten sind für die ambulante vertragsärztliche Versorgung in Arztpraxen keine einrichtungsbezogenen Qualitätsberichte im SGB V gesetzlich vorgeschrieben. Somit bleibt es den einzelnen Versorgern überlassen, welche Daten erfasst, verarbeitet und offentlich zugänglich gemacht werden.

Aufgrund ihrer Zuständigkeiten hinsichtlich der Qualitätssicherung, beispielsweise sind hier Zugangsvoraussetzungen wie Facharztstatus, Verpflichtung zur Fort- bildung und Genehmigungsvorbehalt zu nennen, veröffentlicht die Kassenärztliche Bundesvereinigung (KBV) regelmäßig Qualitätsberichte. Der Qualitätsbericht der KBV beinhaltet aggregierte Daten auf Ebene der kassenärztlichen Vereinigungen sowie für die verschiedenen Fachbereiche. Bezogen auf die Onkologie zählt die KBV allein für das Berichtsjahr 2018 insgesamt 3897 Ärztinnen und Ärzte mit Genehmigung in der onkologischen vertragsärztlichen Versorgung, bei denen $645(16,5 \%)$ arztbezogene Stichproben- bzw. Dokumentationsprüfungen und 12.900 patientenbezogene Dokumentationsprüfungen sowie 3195 Prüfungen von Fortbildungsnachweisen durchgeführt wurden [17]. Informationen für Patientinnen und Patienten bezüglich einzelner Einrichtungen lassen sich hieraus jedoch nicht ableiten. Es bestehen vereinzelt Bestrebungen zur Erstellung praxisbezogener Qualitätsberichte. Diese Überlegungen konnten sich jedoch bislang nicht flächendeckend durchsetzen. Dennoch bleibt die Generierung praxisindividueller Qualitätsberichte für viele Akteure im Gesundheitswesen ein erstrebenswertes Ziel für die interne und externe Qualitätssicherung.
Eine Besonderheit in der ambulanten Versorgung ist die Initiative des Berufsverbands der Niedergelassenen Hämatologen und Onkologen in Deutschland e.V. (BNHO) und dessen wissenschaftlichen Instituts (WINHO), regelmäßig Qualitätsberichte bereitzustellen. Seit 2004 werden in diesen Berichten Daten zu verschiedenen Merkmalen der Versorgung in den Schwerpunktpraxen systematisch erfasst, ausgewertet und dargestellt. Diese Angaben beruhen sowohl auf objektiven Daten, insbesondere KVDT-Daten (Kassenärztliche-Vereinigung-Datentransfer), als auch auf patientenberichteten Angaben zur Versorgung in den Praxen. Darüber hinaus werden Ergebnisse von Mitarbeiterbefragungen sowie projektbezogene Ergebnisse dargestellt [20].

\section{Strukturmerkmale hämato- onkologischer Praxen}

Basierend auf den Daten der Qualitätsberichte lässt sich so u. a. die Entwicklung der Patientenversorgung im niedergelassenen Bereich über mehrere Jahre hinweg darstellen. Dabei ist im Verlauf der Jahre ein Anstieg der Patientenzahl zu verzeichnen (ब Abb. 1). Der durch- 


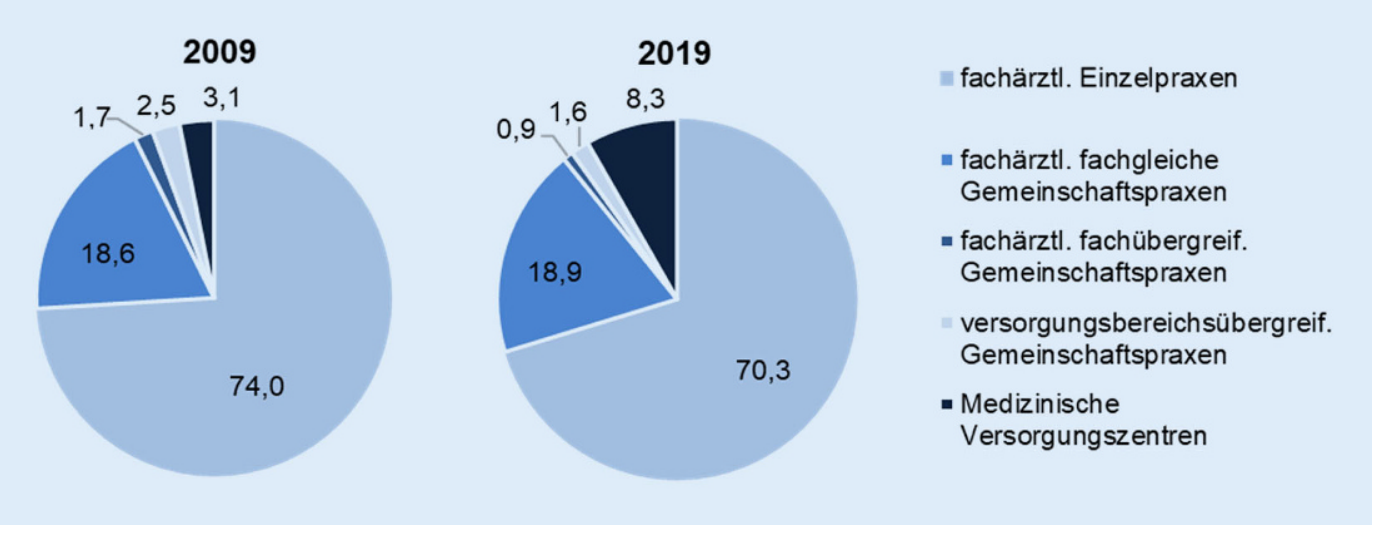

Abb. $2 \triangleleft$ Vergleich der prozentualen Anteile der verschiedenen Formen fachärztlicher Praxen 2009 und 2019. KBV Kassenärztliche Bundesvereinigung, MVZ Medizinisches Versorgungszentrum. (Quelle: Statistische Informationen aus dem Bundesarztregister und MVZ-Statistik der KBV; eigene Darstellung) schnittliche Anteil an Patientinnen und Patienten mit einer Krebserkrankung (International Classification of Diseases, ICD C00-D48) ist in den hämatoonkologischen Schwerpunktpraxen im Jahresvergleich auf über $80 \%$ gestiegen. Die genaue Anzahl der Patientinnen und Patienten, die insgesamt pro Jahr in den hämatoonkologischen Schwerpunktpraxen in Deutschland behandelt werden, lässt sich derzeit nur schätzen. Zieht man die quartalsbezogenen Angaben der KVDT-Daten für das dritte Quartal 2020 heran, liegt die durchschnittliche Anzahl der in BNHO-Mitgliedspraxen behandelten Patientinnen und Patienten in einem Quartal aktuell bei knapp 1.800 bzw. bei knapp 1.500 Krebserkrankten. Dabei ist zu berücksichtigen, dass (Krebs-)Erkrankte meist über einen langen Zeitraum in den Schwerpunktpraxen versorgt werden, wie es $z$. B. bei hämatologischen Erkrankungen der Fall ist.

\section{॥) Innerhalb der Praxen hat sich die Struktur verändert - zu größeren Praxen mit mehr ärztlichem Personal}

Einhergehend mit dem gestiegenen Versorgungs- und Spezialisierungsbedarf in der ambulanten Hämatoonkologie zeichnet sich eine Veränderung der Praxisstrukturen $\mathrm{ab}$, die sich insbesondere in der Praxisgröße und Form der ärztlichen Kooperation widerspiegelt [13]. Besonders hervorzuheben ist hierbei die Entwicklung der Medizinischen Versorgungszentren (MVZ; - Abb. 2). Im Jahr 2019 betrug der Anteil der MVZ in vertragsärztlicher Trägerschaft $42,9 \%$ gegenüber $44,9 \%$ Krankenhaus-MVZ und 16,3\% mit sonstiger Trägerschaft, wobei Mehrfachträgerschaften möglich sind [16]. Aber auch innerhalb der Praxen hat sich die Struktur verändert - hin zu größeren Praxen mit mehr ärztlichem Personal. So ist beispielsweise innerhalb von 5 Jahren der Anteil der Einzelpraxen mit angestellten Ärztinnen bzw. Ärzten von 11,7 im Jahr 2014 auf 17,9 im Jahr 2019 gestiegen [16]. Inwieweit sich die Trägerschaft eines MVZ und die Veränderungen der Praxisstrukturen insgesamt auf die Qualität der ambulanten hämatoonkologischen Versorgung auswirken, ist bislang noch unklar.

\section{Strukturdatenerhebung 2019}

Als Ergänzung zu den Angaben des Qualitätsberichts der hämatoonkologischen Schwerpunktpraxen wurde 2019 eine Strukturdatenerhebung unter den Mitgliedspraxen des BNHO durchgeführt. Hierzu wurden 372 hämatoonkologische Schwerpunktpraxen, darunter auch Zweigpraxen, mit jeweils mindestens einem Mitglied im BNHO gebeten, Angaben zu Praxismerkmalen, Fortbildungsbedarf, Serviceangeboten, QM, Vernetzung sowie weiteren berufspolitischen Themen zu machen. Nach wiederholten Aufforderungen über verschiedene Medien haben sich insgesamt 130 Praxen an der Befragung beteiligt. Die Daten wurden online über eine Eingabemaske erfasst und auf einem gesicherten Server gespeichert. Die gewonnene Stichprobe repräsentiert gut ein Drittel aller BNHO-Mitgliedspraxen. Im Folgenden werden strukturelle Qualitätsmerkmale, die verwendeten QMSysteme sowie die Zusammenarbeit mit zertifizierten Zentren und die Teilnahme an Tumorkonferenzen und klinischen Studien betrachtet.
Die Hälfte der Schwerpunktpraxen, die sich an der Strukturdatenerhebung beteiligt haben, sind Praxis- oder (überörtliche) Berufsausübungsgemeinschaften, nur etwa $17 \%$ der Praxen werden als Einzelpraxen geführt (• Tab. 1). Im Vergleich zur Gesamtheit der BNHO-Mitgliedspraxen sind in der Stichprobe Einzelpraxen etwas unterrepräsentiert und Praxisgemeinschaften sowie (überörtliche) Berufsausübungsgemeinschaften überrepräsentiert. Ähnlich wie die Verteilung der BNHO-Mitgliedspraxen es erwarten lässt, sind knapp $15 \%$ der befragten Praxen inhabergeführte MVZ. Bei der Strukturdatenerhebung des WINHO im Jahr 2014 lag die Anzahl der MVZ noch bei $12 \%$ [19]. Die generelle Zunahme der MVZ spiegelt sich somit auch hier wider. Die durchschnittliche Anzahl der Ärztinnen und Ärzte in den befragten Praxen liegt bei 3,6, wobei der Durchschnitt bei den Einzelpraxen bei 1,7 liegt, bei den Praxisgemeinschaften bzw. (überörtlichen) Berufsausübungsgemeinschaften bei 3,7 und bei den MVZ bei 5,2 .

\section{Verwendete Qualitätsmanage- mentsysteme und Zertifizierungen}

Ein einrichtungsinternes QM ist in der vertragsärztlichen Versorgung nach $\S 135 \mathrm{a}$ Abs. 2 SGB V Pflicht (s. oben). Dabei besteht zwar keine gesetzliche Verpflichtung zur Zertifizierung der Praxen, dennoch lassen sich viele hämatoonkologische Schwerpunktpraxen zertifizieren. Von den befragten Praxen der Strukturdatenerhebung wurden etwas über $60 \%$ mindestens einmal zertifiziert. Eine Rezertifizierung fand bei genau $60 \%$ dieser Praxen statt, wobei über die Hälfte der zertifizierten 
Tab. 1 Ärztliche Kooperationsform bzw. Praxistyp

\begin{tabular}{|c|c|c|}
\hline Praxisart & $\begin{array}{l}\text { Anzahl } \\
n\end{array}$ & $\begin{array}{l}\text { Anteil } \\
(\%)\end{array}$ \\
\hline Einzelpraxis & 22 & 17,2 \\
\hline $\begin{array}{l}\text { Praxisgemeinschaft/ } \\
\text { Berufsausübungs- } \\
\text { gemeinschaft (Ge- } \\
\text { meinschaftspraxis)/ } \\
\text { überörtliche Berufsaus- } \\
\text { übungsgemeinschaft }\end{array}$ & 87 & 68,0 \\
\hline $\begin{array}{l}\text { Medizinisches Versor- } \\
\text { gungszentrum (MVZ) }\end{array}$ & 19 & 14,8 \\
\hline Gesamt & 128 & 100 \\
\hline \multicolumn{3}{|c|}{$\begin{array}{l}\text { WINHO Wissenschaftliches Institut der Nie- } \\
\text { dergelassenen Hämatologen und Onkologen } \\
\text { Quelle: WINHO-Strukturdatenerhebung } 2019\end{array}$} \\
\hline
\end{tabular}

Praxen bereits mehr als 3-mal rezertifiziert wurden. Die am häufigsten in den Praxen verwendeten QM-Systeme sind DIN EN ISO 9001 und Qualität und Entwicklung in Praxen (QEP $\left.{ }^{\circledR}\right)$. Andere QM-Systeme werden deutlich seltener in den hämatoonkologischen Schwerpunktpraxen eingesetzt.

Für die Zertifizierung von Organkrebszentren und Onkologischen Zentren durch die Deutsche Krebsgesellschaft (DKG) sowie von Onkologischen Zentren durch die Deutsche Gesellschaft für Hämatologie und Medizinische Onkologie (DGHO) ist $u$. a. der Nachweis einer Kooperation mit ambulant tätigen Facharztpraxen erforderlich, sodass viele der niedergelassenen Hämatoonkologinnen und Hämatoonkologen mit-zertifiziert sind. Innerhalb dieser Zertifizierungen können hämatoonkologische Praxen ihr eigenes QMSystem verwenden, oder sie übernehmen das implementierte QM-System (KTQ, Kooperation für Transparenz und Qualität im Gesundheitswesen; DIN ISO) des Krankenhauses, mit dem sie kooperieren.

Im Rahmen der Strukturdatenerhebung haben insgesamt 101 Praxen die Frage, ob sie an ausgewiesenen bzw. zertifizierten Zentren in der onkologischen Versorgung als Vertragspartner beteiligt sind, beantwortet. Fast alle Praxen (94\%) gaben an, über mindestens eine Zentrumsbeteiligung zu verfügen. Folglich sind nur $6 \%$ der befragten Praxen nicht mit einem Zentrum verbunden. Im Durchschnitt ist eine hämatoonkologische Schwerpunktpraxis mit 3,55 zertifizierten Zentren vertraglich ver-
Tab. 2 Teilnahme an Tumorkonferenzen

\begin{tabular}{|c|c|c|}
\hline Häufigkeit & $\begin{array}{l}\text { Anzahl } \\
n\end{array}$ & $\begin{array}{l}\text { Anteil } \\
(\%)\end{array}$ \\
\hline $\begin{array}{l}\text { Nicht oder nur sehr } \\
\text { selten }\end{array}$ & 2 & 1,8 \\
\hline Etwa einmal pro Monat & 4 & 3,6 \\
\hline $\begin{array}{l}\text { Etwa jede zweite Wo- } \\
\text { che }\end{array}$ & 8 & 7,1 \\
\hline Etwa jede Woche & 38 & 33,9 \\
\hline $\begin{array}{l}\text { Öfter als einmal pro } \\
\text { Woche }\end{array}$ & 60 & 53,6 \\
\hline Gesamt & 112 & 100 \\
\hline \multicolumn{3}{|c|}{$\begin{array}{l}\text { WINHO Wissenschaftliches Institut der Nie- } \\
\text { dergelassenen Hämatologen und Onkologen } \\
\text { Quelle: WINHO-Strukturdatenerhebung } 2019\end{array}$} \\
\hline
\end{tabular}

bunden (Standardabweichung: 2,22). Diese Zahl ist gegenüber der Befragung von 2014 mit einer durchschnittlichen Zentrumsbeteiligung von 3,3 relativ konstant geblieben [19]. Auffallend ist, dass es regionale Unterschiede zu geben scheint: In einigen Regionen sind Häufungen bei der Anzahl der Praxen ohne Anbindung an ein Zentrum zu verzeichnen, wohingegen in anderen Regionen fast alle der befragten Praxen an mindestens ein Zentrum angebunden sind.

Die Zertifizierung der DGHO als „Kompetenzzentrum für Medikamentöse Tumortherapie" soll ab dem dritten Quartal 2021 zusätzlich zur Verfügung stehen. Das Angebotzur eigenständigen Zertifizierung als „Kompetenzzentrum für Medikamentöse Tumortherapie" richtet sich sowohl an Kliniken als auch an Praxen, sodass sich hämatoonkologische Schwerpunktpraxen auch eigenständig zertifizieren lassen können, ist aber sehr nah an die Kriterien der Zertifizierung der DKG angelehnt [6].

\section{Beteiligungen an Tumor- konferenzen und klinischen Studien}

Die multidisziplinäre Fallbesprechung in Tumorkonferenzen stellt in der Hämatoonkologie seit vielen Jahren einen zentralen Bestandteil der Versorgung von Krebserkrankten dar. Bereits in einer Befragung im Jahr 2010 gaben mehr als $90 \%$ der niedergelassenen Hämatoonkologinnen und Hämatoonkologen an, dass sie regelmäßig an Tumorkonferenzen teilnehmen [12].
Tab. 3 Anteil der Patientinnen und $\mathrm{Pa}$ tienten, die von hämatoonkologischen Schwerpunktpraxen in Tumorkonferenzen eingebracht werden

\begin{tabular}{|l|l|l|}
\hline $\begin{array}{l}\text { Anzahl eigener Patien- } \\
\text { tinnen und Patienten }\end{array}$ & $\begin{array}{l}\text { Anzahl } \\
n\end{array}$ & $\begin{array}{l}\text { Anteil } \\
\text { (\%) }\end{array}$ \\
\hline Keine & 3 & 2,9 \\
\hline Bis zu 5\% & 38 & 37,3 \\
\hline Bis zu 10\% & 28 & 27,5 \\
\hline Bis zu 30\% & 18 & 17,6 \\
\hline Bis zu 50\% & 7 & 6,9 \\
\hline Mehr als 50\% & 8 & 7,8 \\
\hline Gesamt & 102 & 100 \\
\hline $\begin{array}{l}\text { WINHO Wissenschaftliches Institut der Nie- } \\
\text { dergelassenen Hämatologen und Onkologen } \\
\text { Quelle: WINHO-Strukturdatenerhebung 2019 }\end{array}$
\end{tabular}

Demgegenüber sind es in der Strukturdatenerhebung 2019 nur 1,8\% der Praxen, die angaben, dass ihre Ärztinnen bzw. Ärzte nicht oder nur selten an Tumorkonferenzen mitwirken (ब Tab. 2).

\section{I) An Tumorkonferenzen nehmen $90 \%$ der hämatoonkologischen Praxen mindestens einmal pro Woche teil}

Knapp 90\% der befragten Praxen berichten, dass Ärztinnen und Ärzte ihrer Praxis mindestens einmal pro Woche an Tumorkonferenzen partizipieren. Die Erwartung liegt nahe, dass Ärztinnen und Ärzte aus Einzelpraxen insgesamt etwas seltener an Tumorkonferenzen teilnehmen als diejenigen aus größere Praxen, dennoch gaben $45 \%$ der Einzelpraxen an, etwa jede Woche an einer Tumorkonferenz mitzuwirken und $25 \%$ öfter als einmal die Woche. Über alle Schwerpunktpraxen hinweg sind mehr als die Hälfte der Praxen öfter als einmal pro Woche in Tumorkonferenzen eingebunden und ein weiteres Drittel etwa jede Woche.

Mehrheitlich werden Tumorkonferenzen in Krankenhäusern in Präsenzveranstaltungen durchgeführt. Dennoch sind es auch Ärztinnen und Ärzte der hämatoonkologischen Schwerpunktpraxen, die eigene Patientinnen und Patienten in die Tumorkonferenz einbringen (• Tab. 3). Lediglich $3 \%$ der befragten Praxen bringen keine eigenen Patientinnen oder Patienten in Tumorkonferenzen ein.

Insbesondere größere hämatoonkologische Schwerpunktpraxen verfügen über 


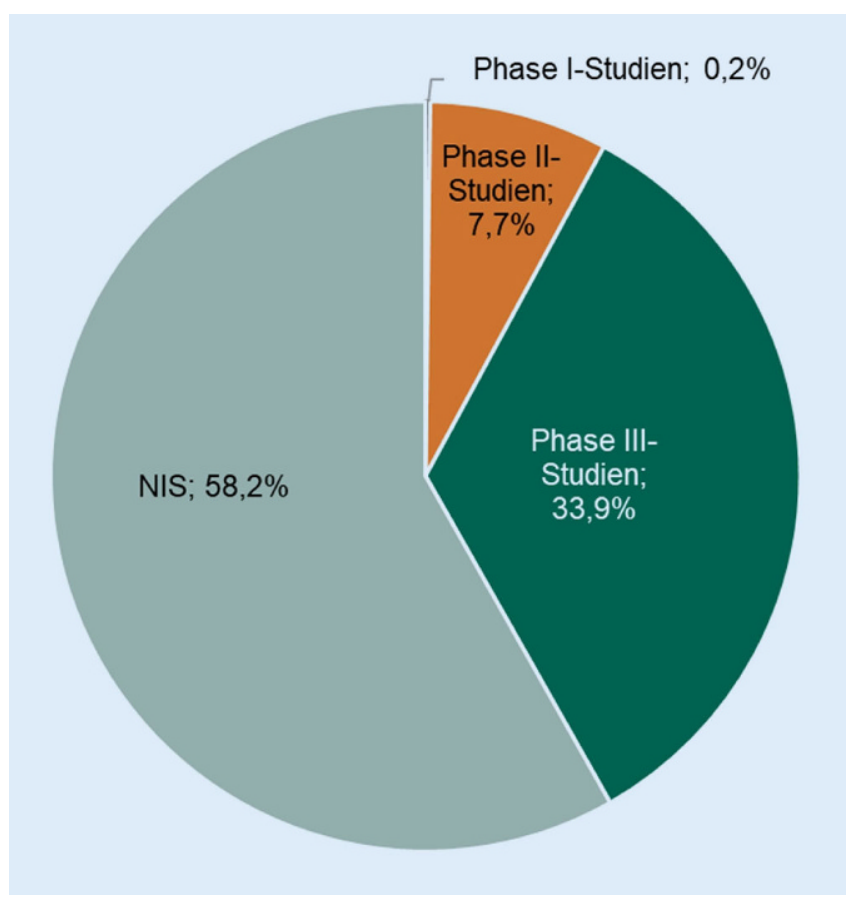

Abb. $3<$ Verteilung der Art der meldepflichtigen Studien in hämatoonkologischen Schwerpunktpraxen im Jahr 2018. NIS Nichtinterventionelle Studien, WINHOWissenschaftliches Institut der Niedergelassenen Hämatologen und Onkologen. (Quelle:WINHOStrukturdatenerhebung 2019) eigene Studienbüros oder angegliederte Studienzentren. In der Strukturdatenerhebung wurde daher die Anzahl der in der jeweiligen Praxis im vorangegangenen Jahr (2018) durchgeführten klinischen Studien erfasst, bei denen Ärztinnen oder Ärzte der jeweiligen Praxis den zuständigen Behörden als Prüfärztin bzw. Prüfarzt gemeldet wurden. Insgesamt haben 93 Praxen hierzu Angaben gemacht, von denen $22 \%$ keine Studien durchgeführt haben. Mehr als $50 \%$ ( $n=47$ Praxen) führten jedoch im Jahr 2018 mindestens 6 verschiedene klinische Studien durch. Knapp 60\% der meldepflichtigen Studien, die im Jahr 2018 in den befragten hämatoonkologischen Schwerpunktpraxen durchgeführt wurden, waren nichtinterventionelle Studien (NIS). Klinische PhaseIII-Studien machten weitere $34 \%$ aus und Phase-II-Studien knapp $8 \%$. Phase-I-Studien wurden in den Schwerpunktpraxen kaum durchgeführt (• Abb. 3).

\section{Diskussion}

Die Ergebnisse der Strukturdatenerhebung 2019 der BNHO-Mitgliedspraxen geben Einblicke in zentrale Merkmale der Strukturqualität, die über die regelmäßig erscheinenden Daten der Qualitätsberichte der hämatoonkologischen Schwerpunktpraxen hinausgehen. Besonders herausstechend ist die starke Vernetzung der Schwerpunktpraxen, die sich u. a. an der engen Bindung mit zertifizierten Zentren, der regelmäßigen Teilnahme an Tumorkonferenzen und der Häufigkeit klinischer Studien in den Praxen zeigt.

\section{॥ Fast alle Praxen sind mit einem ausgewiesenen bzw. zertifizierten Zentrum vertraglich verbunden}

Fast alle Praxen sind mit mindestens einem ausgewiesenen bzw. zertifizierten Zentrum vertraglich verbunden, im Durchschnitt mit mehr als 3 Zentren. Zudem nehmen fast alle Praxen regelmäßig an Tumorkonferenzen teil. Mehr als Dreiviertel der hämatoonkologischen Schwerpunktpraxen haben eigene Ärztinnen oder Ärzte als Prüfärztin bzw. Prüfarzt für klinische Studien angemeldet. Die Ergebnisse der Strukturdatenerhebung basieren auf etwa einem Drittel der Mitgliedspraxen des BNHO. Dies ist zwar für Befragungen in der Versorgungsforschung eine vergleichsweise gute Rücklaufquote, es besteht dennoch eine leichte Verzerrung der Daten, da Einzelpraxen leicht unterund Praxisgemeinschaften bzw. (überörtliche) Berufsausübungsgemeinschaften überrepräsentiert sind.

Regionale Besonderheiten wie z. B. die Verfügbarkeit aller relevanter Partner- einrichtungen für die Zertifizierung eines Netzwerks zur Behandlung von Krebskranken wurden in dieser Auswertung nicht berücksichtigt. Bei der 2012 eingeführten ambulanten spezialfachärztlichen Versorgung (ASV) wird die räumliche Nähe der beteiligten Versorgungseinrichtungen vorausgesetzt. Auch hier wird die Vernetzung in der ambulanten onkologischen Versorgung deutlich. So haben sich beispielsweise seit 2014 mehr als 130 ASVTeams für gastrointestinale Tumoren mit über 7000 Ärztinnen und Ärzten zusammengefunden. So unterstützt die ASV einerseits die interdisziplinäre Vernetzung, andererseits verstärkt sie auch den lokalen Wettbewerb in der ambulanten Versorgung. Die Auswirkungen auf die Qualität der Patientenversorgung lassen sich zum jetzigen Zeitpunkt noch nicht abschätzen [5].

In diesem Beitrag wurde der Schwerpunkt auf einige strukturelle Qualitätsmerkmale gelegt. Mit Blick auf die Einteilung in Struktur-, Prozess- und Ergebnisqualität [8] lässt sich die Strukturqualität der hämatoonkologischen Schwerpunktpraxen gut abbilden. Die Entwicklung und Erfassung von Qualitätsindikatoren zur Abbildung der Prozessqualität stellt ein wichtiges Instrument der Dauerbeobachtung der Versorgungsqualität dar [2]. Doch dies erfordert einen hohen personellen und zeitlichen Aufwand, der im Praxisalltag kaum zu stemmen ist [14]. Die Ergebnisse der ärztlichen Versorgung sind für die behandelnden und die erkrankten Personen und Angehörigen i. d. R. sichtbar. Eine strukturierte und regelhafte Erfassung und Abbildung der Ergebnisqualität unterliegt jedoch ähnlichen Herausforderungen wie die Darstellung der Prozessqualität und kann außerhalb von Studienzentren im Praxisalltag kaum geleistet werden. Bereits die vollständige Meldung detaillierter Daten zu Therapie und Verlauf an die Krebsregister stellt niedergelassene Ärztinnen und Ärzte vor Herausforderungen, die auch in Zukunft weiter angegangen werden müssen, um diese Daten zur Qualitätssicherung besser nutzen zu können [10].

Wichtige Hinweise zur Qualität der Versorgung liefern patientenberichtete Ergebnisse (PRO) und Erfahrungen (PRE). In den jährlichen Befragungen des WINHO 


\section{Infobox 1}

Aktueller Qualitätsbericht der niedergelassenen Hämatologen und Onkologen

- https://dl.winho.de/QS-Bericht_2020_ Web.pdf

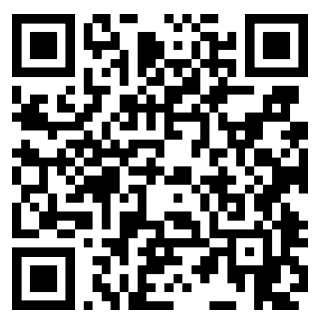

von etwa 10.000 Patientinnen und Patienten in hämatoonkologischen Schwerpunktpraxen zeichnet sich konstant ein positives Bild der Versorgungsqualität bei den niedergelassenen Hämatoonkologinnen und Hämatoonkologen ab [20]. Doch die regelhafte Verknüpfung von strukturellen und prozessualen Qualitätsmerkmalen der hämatoonkologischen Schwerpunktpraxen mit Ergebnisparametern wie Gesamtüberleben, progressionsfreiem Überleben oder auch patientenberichteten Ergebnissen (PRO) zur Analyse und Darstellung der Ergebnisqualität in der ambulanten Versorgung ist nach wie vor ein schwieriges Unterfangen.

Es ist ein schmaler Grat zwischen Maßnahmen zur Qualitätssicherung der Patientenversorgung einerseits und dem administrativen Aufwand, der damit verbunden ist, andererseits. Werden hierdurch personelle und zeitliche Ressourcen zu sehr beansprucht, kann dies ungewollt zulasten der Patientinnen und Patienten gehen, anstatt ihre medizinische Versorgung zu sichern und zu verbessern. Der administrative Aufwand muss letztlich in einem angemessenen Verhältnis zur Effektivität der qualitätssichernden Maßnahme stehen. Dennoch ist die Erfassung und transparente Darstellung von Qualitätskriterien ein wichtiger Indikator für die interne und externe Qualitätssicherung, um zu gewährleisten, dass die Versorgung von Patientinnen und Patienten mit hohen Standards durchgeführt wird.

Die Notwendigkeit, die sektorübergreifende Qualitätssicherung und -berichterstattung weiterzudenken, zeigt sich somit nicht nur in politischen Diskussionen, sondern auch durch die in diesem Beitrag dargestellte Vernetzung ambulanter Ein-

Quality assurance in haemato-oncology specialist practices. Results of a 2019 structural data survey

Background: The regularly published quality reports of the haemato-oncology specialist practices primarily include billing data and results of patient and staff surveys as well as current project-related results. In order to obtain further information on structural quality, an additional survey was conducted among haemato-oncology practices.

Objectives: The goals are the analysis and presentation of structural quality characteristics of haemato-oncology specialist practices.

Methods: We performed an online survey of practices with at least one member in the Professional Association of Office-based Haematologists and Oncologists in Germany in 2019 on practice characteristics, professional policy issues, quality management, and networking.

Results: Of 372 practices contacted, 130 participated in the structural data survey (35\%). Regarding the topics of quality management and networking, the data show that more than $60 \%$ of the practices have a certified quality management system. Almost all practices (94\%) are contracted to at least one certified centre and regularly participate in tumour conferences (98\%). Furthermore, $77 \%$ of the practices participate in clinical trials.

Conclusions: The results of the 2019 structural data survey provide insights into quality characteristics of haemato-oncology specialist practices and demonstrate a strong cross-sector networking of these practices in Germany.

\section{Keywords}

Quality management · Networking · Certification · Neoplasms · Outpatients

richtungen. Dies gilt umso mehr mit Blick auf die Herausforderungen, die durch die SARS-CoV-2-Pandemie entstanden sind [11], als auch hinsichtlich der Einbindung der Molekulardiagnostik, innovativer therapeutischer Möglichkeiten, der wissensgenerierenden Versorgung oder der wohnortnahen Langzeitversorgung [15].

\section{Fazit für die Praxis}

- Die Anzahl der Patientinnen und Patienten, die in hämatoonkologischen Schwerpunktpraxen versorgt werden, ist in den letzten Jahren weiter gestiegen.

- Fast alle hämatoonkologischen Schwerpunktpraxen sind mit mindestens einem Zentrum vertraglich verbunden, im Durchschnitt mit mehr als 3 Zentren.

- Fast alle hämatoonkologischen Schwerpunktpraxen nehmen regelmäßig an Tumorkonferenzen teil.

- Mehr als Dreiviertel der hämatoonkologischen Schwerpunktpraxen haben eigene Ärztinnen oder Ärzte als Prüfärztin bzw. Prüfarzt für klinische Studien angemeldet.

- Die starke Vernetzung der Praxen verdeutlicht die Notwendigkeit der Weiterentwicklung der sektorübergreifenden Qualitätssicherung und -berichterstattung.

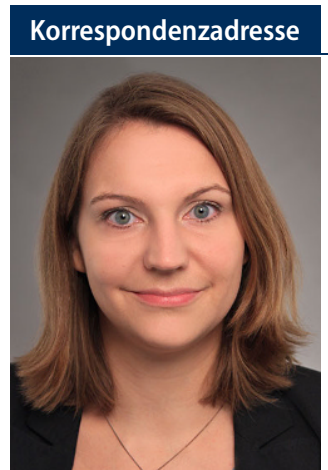

Dr. Kerstin Hermes-Moll

Wissenschaftliches Institut der Niedergelassenen Hämatologen und Onkologen (WINHO)

Vor den Siebenburgen 2, 50676 Köln, Deutschland

hermes-moll@winho.de

\section{Einhaltung ethischer Richtlinien}

Interessenkonflikt. K. Hermes-Moll, T. Walawgo, S. Osburg und V. Heidt geben an, dass kein Interessenkonflikt besteht.

Für diesen Beitrag wurden von den Autoren keine Studien an Menschen oder Tieren durchgeführt. Für die aufgeführten Studien gelten die jeweils dort angegebenen ethischen Richtlinien. 


\section{Literatur}

1. Aly A-F, Schmitz S (2005) Qualitätssicherung in der ambulanten onkologischen Krankenversorgung. Onkologe 11:1278-1285

2. Baumann W, Buschmann-Maiworm R, Schmitz $S$ (2012) Qualitätssicherung und Qualitätsindikatoren. Onkologe 18:134-141

3. Baumann W, Schmitz S (2016) Entwicklungen in der onkologischen Versorgung. Ambulant und stationär. Onkologe 3:158-166

4. Baumann W, Steinmetz HT (2010) Qualitätsmanagement in der ambulanten Versorgung onkologischer Patienten. In: Schmalenberg H, Hartmann R, Baumann W (Hrsg) Qual. Zertif. Onkol. Springer, Berlin, Heidelberg, S77-106

5. Dengler R (2021) Ambulante spezialfachärztliche Versorgung: Steht der Patient im Mittelpunkt? GGW 21:15-23

6. Deutsche Gesellschaft für Hämatologie und Medizinische Onkologie (DGHO) (2020) Checkliste für die Zertifizierung von Kompetenzzentren für Medikamentöse Tumortherapie. Version 1.0. https://www.onkologie-zertifizierung.de/web/ verfahrensanweisungen-und-formulare/copy2 of_DokCLKMTV1.0.pdf.Zugegriffen: 9. März 2021

7. Diel F (2015) Qualitätsförderung und Qualitätssicherung in der ambulanten Versorgung. Gesundh Sozialpolit 69:34-40

8. Donabedian A (1966) Evaluating the quality of medical care. Milbank Mem Fund Q 44:166-203

9. Gemeinsamer Bundesausschuss (G-BA) Richtlinie des Gemeinsamen Bundesausschusses über grundsätzliche Anforderungen an ein einrichtungsinternes Qualitätsmanagement für Vertragsärztinnen und Vertragsärzte, Vertragspsychotherapeutinnen und Vertragspsychotherapeuten, medizinische Versorgungszentren, Vertragszahnärztinnen und Vertragszahnärzte sowie zugelassene Krankenhäuser. https://www. g-ba.de/richtlinien/87/Zugegriffen 10. April 2021

10. Gurung-Schönfeld I, Kraywinkel K (2021) Krebsregistrierung heute: zwischen Epidemiologie, Qualitätssicherung und Forschung. Epid Bull 4:3-9. https://doi.org/10.25646/7847

11. Heidt $V$, Knauf W, Illmer T et al (2021) Hämatoonkologische Praxen. Trotz Pandemie ambulant gut versorgt. Dtsch Arztebl 118:A310-A313

12. Hermes-Moll K, Dengler R, Riese C, Baumann W (2016) Tumor boards from the perspective of ambulant oncological care. Oncol Res Treat 39:377-383

13. Hermes-Moll K, Heidt V (2019) Wandel der ambulanten onkologischen Versorgung und die Folgen. InFo Hämatol Onkol 22:68-71

14. Hermes-Moll K, Klein A, Schmitz S, Baumann W (2020) Ergebnisse einer Pilotstudie zur Erfassung von Qualitätsindikatoren in der ambulanten onkologischen Versorgung. Gesundheitswesen 82:63-71

15. Hermes-Moll K, Osburg S, HeidtV (2020) Patientenorientierung bei zunehmender Komplexität. InFo Hämatol Onkol 23:12-15

16. KBV - Kassenärztliche Bundesvereinigung (2014) Statistische Informationen aus dem Bundesarztregister. Bundesgebietinsgesamt

17. KBV - Kassenärztliche Bundesvereinigung (2019) Qualitätsbericht 2019/Berichtsjahr 2018. KBV, Berlin

18. Schaefer C (2019) Ambulante ärztliche Qualitätssicherung: Welche Rolle spielen Leitlinien? Forum 34:269-273

19. Wissenschaftliches Institut der Niedergelassenen Hämatologen und Onkologen (2015) Qualitätsbe- richt der onkologischen Schwerpunktpraxen 2014. Wissenschaftliches Institut der Niedergelassenen Hämatologen und Onkologen, Köln

20. Wissenschaftliches Institut der Niedergelassenen Hämatologen und Onkologen (2020) Qualitätsbericht der hämatologischen und onkologischen Schwerpunktpraxen 2019. Wissenschaftliches Institut der Niedergelassenen Hämatologen und Onkologen, Köln

\section{Hier steht eine Anzeige. Springer}

